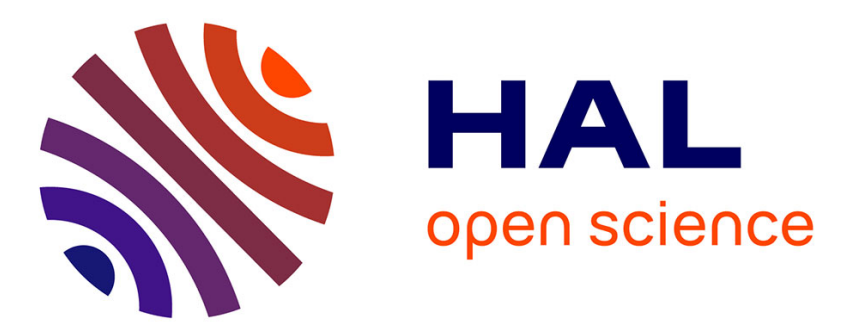

\title{
An Assessment Methodology of Smartphone Positioning Performance for Collaborative Scenarios in Urban Environment
}

Thomas Verheyde, Antoine Blais, Christophe Macabiau, François-Xavier Marmet

\section{- To cite this version:}

Thomas Verheyde, Antoine Blais, Christophe Macabiau, François-Xavier Marmet. An Assessment Methodology of Smartphone Positioning Performance for Collaborative Scenarios in Urban Environment. ION GNSS+ 2020, 33rd International Technical Meeting of the Satellite Division of the Institute of Navigation, Sep 2020, Virtual event, United States. pp 1893 - 1901, 10.33012/2020.17626 . hal02964014

\section{HAL Id: hal-02964014 \\ https://hal-enac.archives-ouvertes.fr/hal-02964014}

Submitted on 27 Nov 2020

HAL is a multi-disciplinary open access archive for the deposit and dissemination of scientific research documents, whether they are published or not. The documents may come from teaching and research institutions in France or abroad, or from public or private research centers.
L'archive ouverte pluridisciplinaire HAL, est destinée au dépôt et à la diffusion de documents scientifiques de niveau recherche, publiés ou non, émanant des établissements d'enseignement et de recherche français ou étrangers, des laboratoires publics ou privés. 


\title{
An Assessment Methodology of Smartphones Positioning Performance for Collaborative Scenarios in Urban Environment
}

\author{
Thomas Verheyde, TéSA -Telecommunication Research Laboratory, France. \\ Antoine Blais, ENAC - École Nationale de l'Aviation Civile, Université de Toulouse, France. \\ Christophe Macabiau, ENAC-École Nationale de l'Aviation Civile, Université de Toulouse, France. \\ François-Xavier Marmet, CNES - Centre National d'Études Spatiales, France.
}

\section{BIOGRAPHIES}

Thomas VERHEYDE graduated from ENAC ("The French civil aviation university") with a Master of Science in Aerospace Systems Navigation \& Telecommunication. Currently pursuing my PhD in the GNSS domain, my thesis' title is "Precise Cooperative Positioning of Low-Cost Mobiles in an Urban Environment". Employed by TéSA (Telecommunication Research Laboratory) in Toulouse and my research is co-funded by CNES (French space agency) and Thalès Alénia Space.

Dr. Antoine BLAIS has earned an engineering degree from ENAC (École Nationale de l'Aviation Civile, the French Civil Aviation University), Toulouse, France, in electrical engineering in 1993, a Master of Science in Signal, Image and Acoustics from the Institut National Polytechnique de Toulouse (INPT), France, in 2003 and a Ph.D. degree from INPT in 2014. Antoine Blais is currently an assistant professor in the Telecommunication Research Team of ENAC. His research activities relate to Global Navigation Satellite System (GNSS) receiver signal processing and Software Defined Radio (SDR) in particular.

Dr. Christophe MACABIAU graduated as electronics engineer in 1992 from the ENAC (École Nationale de l'Aviation Civile) in Toulouse, France. Since 1994, he has been working on the application of satellite navigation techniques to civil aviation. He received his $\mathrm{PhD}$ in 1997 and has been in charge of the signal processing lab of ENAC since 2000, where he also started dealing with navigation techniques for urban navigation. He is currently the head of the TELECOM lab of ENAC, which includes research groups on signal processing and navigation, electromagnetics and data communication networks.

Francois-Xavier MARMET graduated from ENAC (the French Civil Aviation University) in 2011. He has been with CNES (the French Space Agency) since 2013 where he is involved in spaceborne GNSS receivers' development, niche PNT systems design and performance assessment, and Galileo design and coordination working groups.

\section{ABSTRACT}

The release of Android Global Navigation Satellite Systems (GNSS) raw measurements in late 2016 unlocked the access of smartphones' embedded positioning chipset capabilities for developers and the scientific community. This groundbreaking announcement was followed by technical innovations, made by smartphone brands and chipset manufacturers, in order to obtain the world's most precise smartphone on the market. In recent years, several studies investigated the development of advanced positioning techniques (e.g. Precise Point Positioning (PPP), Real-Time Kinematic (RTK)) using Android raw data measurements. However, most studies drawn their conclusions based on one smartphone brand and model in optimal open-sky conditions despite the fact that most smartphone-based positioning activities are achieved in urban and sub-urban areas. In order to overcome urban smartphone-based positioning issues, we ambition to develop a collaborative user's network taking advantage of the tremendous numbers of connected Android devices in today's busy city centers.

A throughout study has been conducted in the city center of Toulouse in France for characterizing smartphone positioning performance in both nominal and urban conditions. Various limiting factors were exposed during our data collection campaign. Nevertheless, the investigation conducted on Android GNSS raw measurement uncovered smartphone positioning potential for navigation applications in constraint environment. A methodology assessment has been implemented in order to identify, 
characterize and compare smartphones' positioning performances. A classification of key parameters has been determined focusing on the implementation of collaborative algorithms, revealing the attributes and components for smartphone-based collaborative methods.

Thereafter, a comprehensive state of the art review on existing cooperative positioning techniques, has been achieved. An evaluation of the feasibility and the applicability of those methods into the smartphone domain has been made. We present a method based on simple assumptions, without third-party equipment and data, only relying on smartphones' own data combination. Our cooperative network can be described as a low-cost embedded structure aiming at providing positioning assistance to its users.

\section{INTRODUCTION}

In May 2016, Google announced during their I/O conference that GNSS raw data measurements would be accessible on any Android devices running Android Nougat (7.0) and above. This announcement unlocked opportunities, for developers and the scientific community, to capitalize on the embedded positioning chipset inside their smartphone. This trend reached chipsets manufacturers that engaged into a tremendous technological race for developing the most precise and accurate location device. Technological innovations made multi-constellation and multi-frequency smartphones a reality.

In parallel, several studies investigated the development of advanced positioning techniques (e.g. Precise Point Positioning (PPP), Real-Time Kinematic (RTK)) using Android raw data measurements [1]. Results showed that precise smartphone-based positioning was feasible despite the use of a linearly polarized antenna due to the tight phones' hardware architecture [2].

Comparisons between commercial-off-the-shelf (COTS) receivers and Xiaomi smartphones testified about the level of performance that we can expect from smartphones' embedded chipset [3]. However, most studies drawn their conclusions based on one smartphone brand and model in optimal open-sky conditions. Although, most smartphone-based positioning activities are achieved in urban and sub-urban areas. In urban conditions, signals are degraded from disruptive multipath and non-lineof-sight (NLOS) interferences that represent the main challenge associated with urban positioning. Apprehending those difficulties become even more challenging when using a low-grade smartphone antenna. In order to overcome urban smartphone-based positioning issues, we ambition to develop a collaborative user network taking advantage of the tremendous numbers of connected Android devices in today's busy city centers.

This paper will be articulated around two axes. A methodology assessment will be presented, in first place, describing smartphone positioning performance parameters in both nominal and urban environments. Our analysis aims at comparing different Android smartphones pedestrian-based positioning capabilities in different constrained environments. A methodology will be developed for characterizing any Android device.

Following this study, a review of the state of the art regarding collaborative positioning will be made. This literature review will guide our research toward the cooperative network that we envision.

\section{ASSESSMENT METHODOLOGY}

Since the release of Android Nougat (7.0), users are able to record GNSS raw data measurements from mass market Android devices. The main advantage foreseen by Google for this implementation was for developers and the scientific community to design and implement advanced positioning algorithms. However, the GNSS receiver architecture on the different models of smartphone's embedded chipset are unknown. The white paper on Android GNSS raw data measurements written by the "raw measurements task force" commissioned by the European Global Navigation Satellite Systems Agency (GSA) made an analogy between smartphone's receiver and regular GNSS receiver. In their paper [4], they specified that the signal processing block is software-based and is not designed to operate specifically on mass market mobile design. Considering the wide variety of Android smartphones, the integrated navigation chipset has been created to act as a black box only output ting PVT results and more recently GNSS raw data measurements.

In order to overcome the ambiguity generated by Android's black box processes for recording GNSS raw data measurements, we studied the implementation of an assessment methodology. Our objective was to better cope with those raw measurement and better understand their impact on the final positioning solution while navigating deep urban environment.

Our analysis method starts with the description of our experiment protocol that took place in the packed city center of Toulouse. Thereafter, smartphones positioning performance will be characterized by modeling receiver main parameters in nominal, open-sky conditions. Finally, a study of tracked signals for our tested smartphones will be made in multiple urban scenarios. An emphasis on sharable data and measurements will be given in consideration of the implementation of a collaborative smartphone network. 


\section{II.1 - Experiment Protocol}

Our data collection campaign took place in the heart of the city center of Toulouse in France. The trajectory chose for that day depicted on Figure 1. This trajectory has been selected for its versatility in terms of reception conditions and constraints that could be found in modern city centers. Along the way, collaborative scenarios were implemented in order to better represent smartphone-based pedestrian navigation in urban areas. Those scenarios are represented by letters on figure 1 .

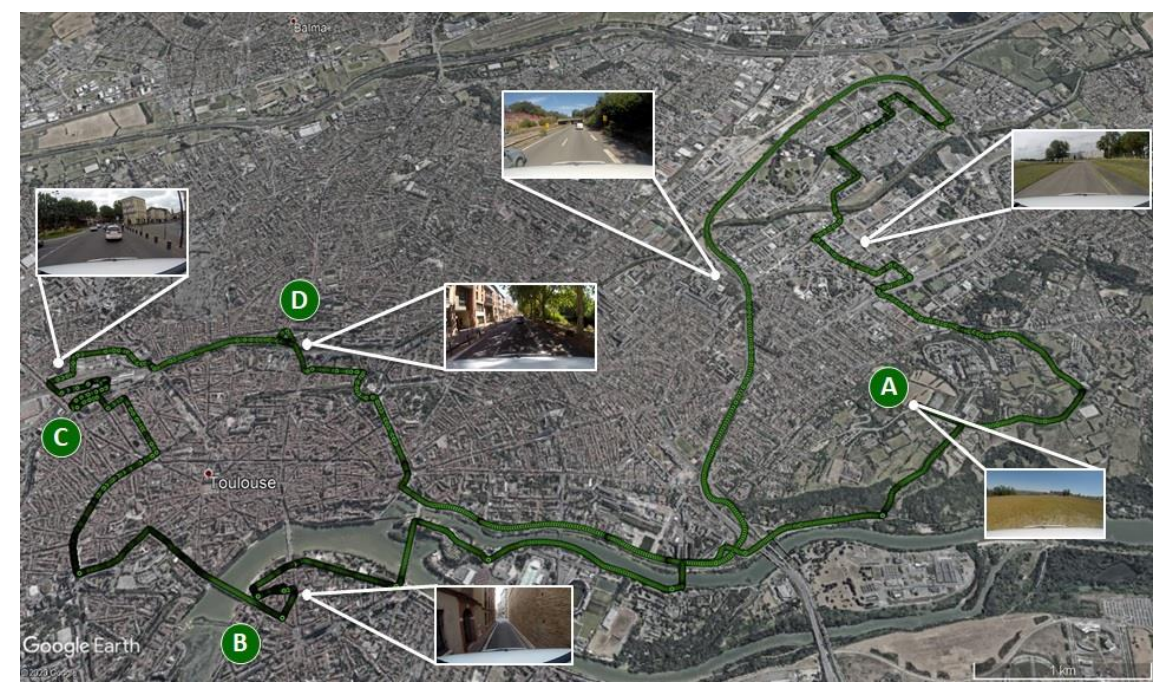

Figure 1: Data Collection Campaign Trajectory \& Scenarios - Toulouse, 2019, 6th August.

Figure 2 is the visual representation of the wide array of tested smartphone during this data campaign. The models tested were all operating Android Q (10.0). The selected smartphones represent the variety of brands and models that can be found in everyone's pocket. Following the same diversity principle, we opted for smartphone operating on different Central Processing Unit (CPU) chipset from the three main manufacturers. All tested devices were multi-constellation and multi-frequency GNSS receivers, only the Google Pixel 3 was single frequency.

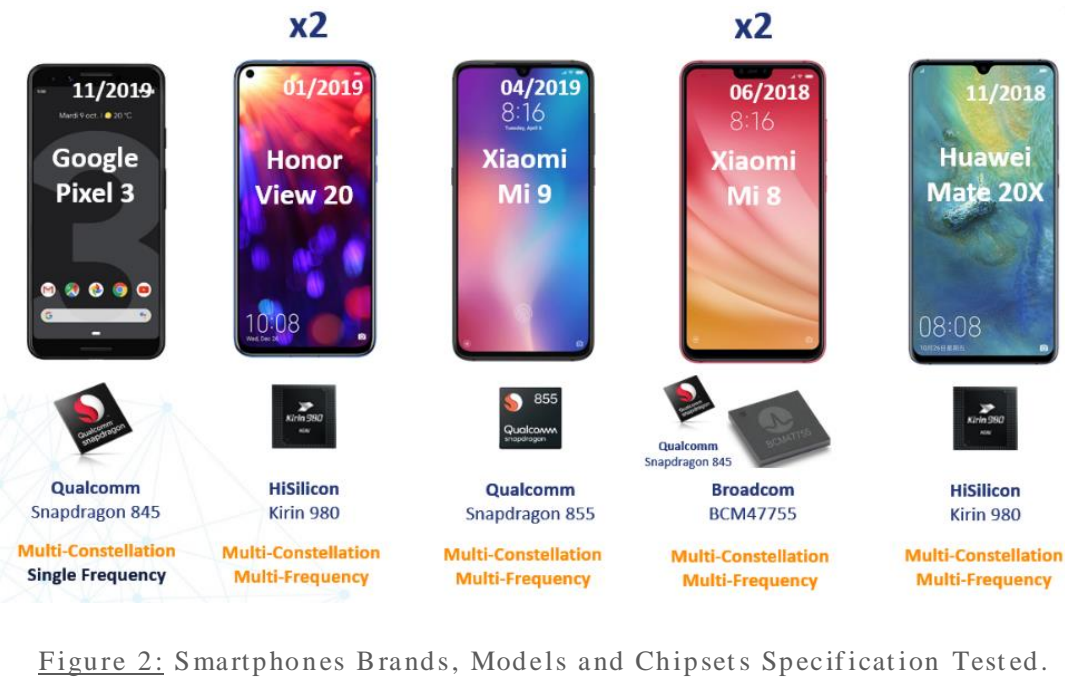

Two research vehicles were used during this experimentation. The goal of using two cars was to simulate two different users in a collaborative setting. The smartphones were splited into two groups and placed on the roof of each vehicle. Inside each car, COTS high end GNSS receivers were set for reference purposes. The data collection campaign lasted for more than two hours. 


\section{II.2 - Nominal Conditions}

We started by analyzing smartphone positioning performance parameters in open-sky conditions. This primary study would help us understand the nominal performances of each device. A correlation between smartphone brands, models and chipset characteristics was also of interest. A preliminary study made by Lethova. et al. [5], compared smartphones performance data to COTS receivers.

In this analysis, we identified the four main parameters designated as performance parameters:

- $\quad$ Phase jitter $\left(\sigma_{P L L}\right)$

- $\quad$ Code jitter $\left(\sigma_{D L L}\right)$

- Doppler jitter $\left(\sigma_{F L L}\right)$

- $\quad$ Clock drift $(c \dot{\partial} t)$

These four parameters were successfully estimated and modelled. The extensive analysis and results were presented during the International Navigation Conference (INC) 2019 [6].

According to our findings, the "lowest-cost GNSS receivers" segments represented by smartphones seems to be as efficient as standard low-cost GNSS receivers (e.g. Ublox M8T).
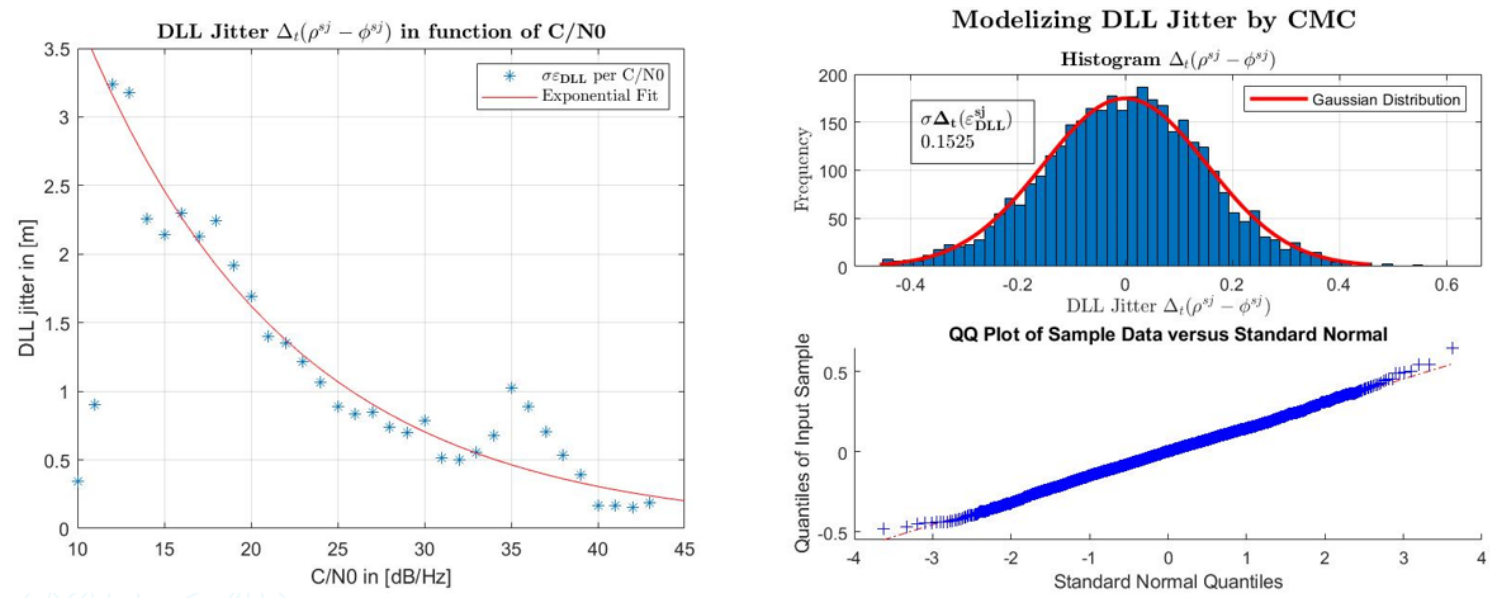

Figure 3: DLL Jitter Estimation in Function of C/NO \& Histogram for Honor View 20 [6].

Error levels estimated for smartphones were of the same order of magnitude for all tested devices. A certain exception has to be noted, smartphone that could not record phase measurements were showing higher levels of error. Figure 3 shows the modelling and the results obtained for the Honor View 20.

It was concluded that smartphone positioning performance parameters are not necessarily limiting factors for smartphone-based positioning and navigation. Therefore, the implementation of smartphone collaborative positioning algorithm can be planned.

\section{II.3 - Urban Environment}

Urban environment positioning constitutes a great challenge for non-aided GNSS receivers. Signals can easily get degraded either by disruptive multipath and by NLOS signals reception. Those disruptions are mainly due to environment around the user made of tall buildings and usually referred as "urban canyons". These limiting factors are aggravated by smartphone's components. Indeed, the linearly polarized patch antenna, mounted on a tight logic board, inside our smartphones, is not optimized for acquiring circularly polarized GNSS signals in disruptive conditions.

Our analysis aims at recognizing potential limiting factors for developing a smartphone collaborative system. The first observation that came from our data collection campaign was the unreliability of phase measurements. Certain recent smartphones (e.g. Samsung S10, Xiaomi Mi9 ...) do not record any phase measurements whereas older version of these phones did record this type of data. Up to this date, we do not know if phase measurements recording will become the norm for Android GNSS raw data measurements. Secondly, we consider using phase measurements could become deceitful since numerous cycle slip occurrences were observed on every tested smartphone. In average, considering all our smartphones, more than 500 occurrences happened over the course of the data collection campaign (2h10min). As an example, red dots on Figure 4 represent cycle slip events for a Xiaomi Mi 8 in urban conditions. 


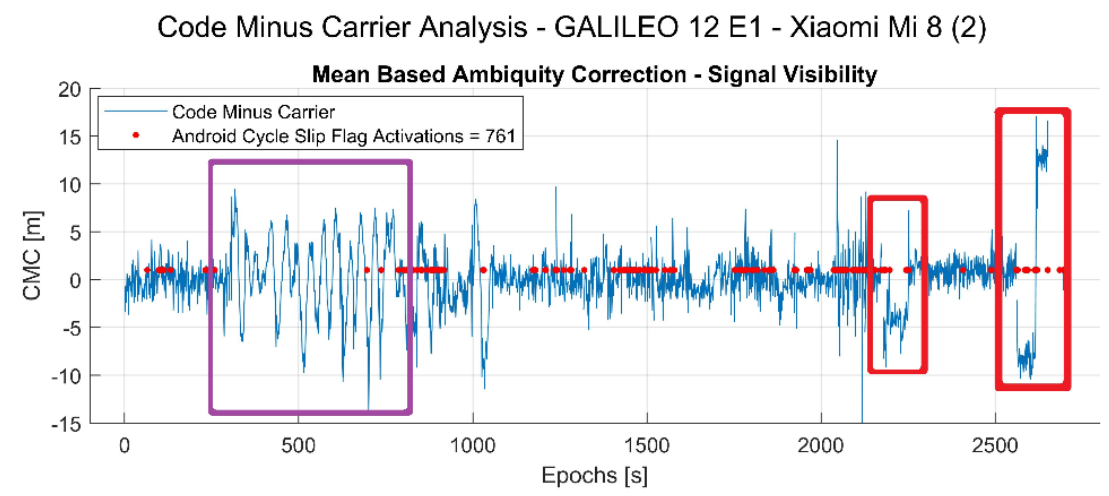

Figure 4: Analysis of the CMC for Galileo PRN12 E1 for a Xiaomi Mi8 in urban constraints [7].

Android GNSS raw data measurements provide flags mechanisms for multipath and cycle slip events. However, it appears that their detection algorithms are unknown. As a part of our urban analysis, we conducted a study on those flags' detection mechanisms. The results provided in [7] reveal that no raw data measurements can be directly correlated to the output value of those flags. A more complex algorithm is then responsible for determining cycle slip and multipath events. The detection accuracy of cycle slip and multipath flags was also studied. It showed that multipath detection mechanisms are inconsistent and could not detect "textbook" multipath events. On the other side, cycle slip detection proved to be more effective, even if we can regret the high false alarm detection rate $\left(P_{F A}\right)$ which makes this parameter incompatible with collaborative positioning when used as is.

Smartphone positioning in constrained environments turned out to be more accurate than expected. Most of the time, 3D position error compared to a high-end COTS receiver (NovAtel SPAN: An IMU/GNSS coupled receiver) was below 5 meters. This is only true if we look at the position given directly by the phone outputted by the Fused Location Provider (FLP) Android's algorithm. This algorithm is often described as a tight integration design between cellular, Wi-Fi, Bluetooth and the Inertial Measurement Unit (IMU) data [4]. During our test campaign we observed that the embedded GNSS receiver has a tendency to favor certain signals compare to others. It seems that a weighting process is made by the FLP as well depending on the signal's constellation. For example, for the L5 frequency, most of the received signals are from the Galileo constellation. This ascertainment is validated by the data analyzed on Figure 5. The percentages showed on the figure correspond to the ratio between the L1 and L5 frequency for GPS and Galileo. For some smartphones such as the Honor View 20, the proportion of L5 signals tracked is up to $92 \%$ for Galileo

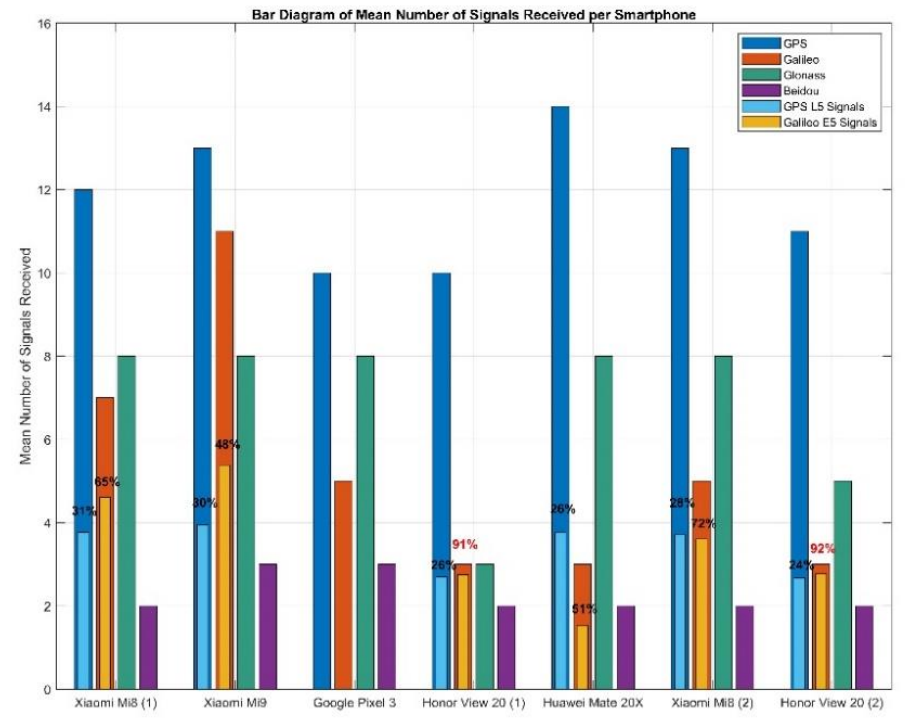


For each frequency, signals from the best deployed constellations - and from the most mature ones in case several are well deployed - seems to be privileged by the GNSS software developed by chipset manufacturers embedded on smartphones.

Signal availability in city center was higher than expected. More than 30 signals were tracked in average from our fleet of tested smartphones. The surprising increase seen on this indicator can be explained by the fact that the phones are now multiconstellation and multi-frequency which obviously increase the number of tracked signals. Moreover, it appears that signals are acquired and tracked down to very low C/NO. Up to $10 \%$ of tracked signals have a C/N0 below of $15 \mathrm{dBHz}$.

The presented throughout analysis allowed us to better understand smartphones' integrated chipset "black box" processes. The characterization methodology set during our data campaign analysis enlighten differences in positioning performance between different smartphones. The estimation of key positioning performance parameters allowed us to quantify those differences for both nominal and urban environments. Phones positioning capabilities disparity makes us believe that a collaborative system can be put in place to improve smartphones-based positioning in constraint environment.

\section{SMARTPHONE COLLABORATIVE TECHNIQUES}

The implementation of a user-oriented network requires a rigorous definition of our intended collaborative system. Cooperative algorithms have been studied extensively in the literature answering problematics linked with deep urban environment navigation, indoor positioning and more recently for autonomous driving. Based on a detailed state of the art review, we will describe collaborative methods that could be applied in a smartphone-based cooperative network. The very first step toward the creation of a network is to establish the goal and functionality of our system. Various cooperative solutions will be studied and an analysis of their applicability to the smartphone positioning domain will be provided.

\section{III.1 - State of the Art Review}

\section{III.1.1 - Definition}

In their paper [8], Garello et al. drew the foundations of what constitutes a cooperative network. A collaborative network is characterized by several GNSS receivers, also referred to as users, communicating with each other via a communication system. Users should be able to exchange data with the intention to aid one or more users. The sharing of key parameters across a cluster of GNSS receivers allows faster positioning computations and the development of complex navigation algorithms.

A collaborative positioning network can be defined by three key characteristics:

- Network architecture

- Nature of the exchanged data

- Implementation methods

- Network Architecture: The definition of an architecture for our network corresponds to the method that will be used by network's user to communicate with each other. A centralized network architecture made of a centralized processing unit where users exchange data only via this central head system. This option, however, will not be preferred for a smartphonebased network. On the other hand, a Peer-to-Peer (P2P) structure will be favored. This type of network architecture corresponds to a cluster of users exchanging information between each other, the processing power will be provided by users' receivers. A third architecture could be imagined, a structure that combines both previously cited methods. A P2P structure supervised by a central unit for controlling the information freely exchanged by network' users.

- Nature of Exchanged Data: A collaborative network is also characterized by the nature of exchanged data. In order to fully take advantages of smartphones' capabilities, multiple data types will be processed within our system such as: GNSS Android raw data measurements plus additional Signals of Opportunity (SoOP). Those data can be qualified also as a "Hybrid" data exchange. Any data recordable by smartphone (e.g. Wi-Fi RTT, Bluetooth RSS, IMU, 5G) is eligible to be used and exchanged in a smartphone-based cooperative network.

- Implementation Methods: This network characteristic represents where does our collaborative algorithm occur in the positioning estimation process. Therefore, the implementation method chosen for mobile cooperative networking is set by which GNSS parameters are retrieved and used. Android GNSS raw data measurements will be our primary data source exchanged and correspond to the output of the signal processing techniques of any GNSS receivers. Our selected implementation method can be defined as a method operating at the range layer, before computing PVT estimation algorithm. 


\section{III.1.2 - Smartphone-based collaborative network}

The main objective of our smartphone-based collaborative network will be to improve one or more user smartphone's positioning performances. Improving positioning performance can be subject to the enhancement of the four parameters used to describe GNSS receiver performance which are accuracy, availability, continuity and integrity. Working on smartphonebased system introduce various limitation for implementing an efficient and reliable network. Those limitations are seen as a bedrock foundation to our infrastructure and are listed below:

- Controlled communication link: we assume that a controlled communication exists allowing users to exchange data via a safe and fast network. In other terms, we assume that the communication link ensures the integrity of exchanged data packets and prevents data packets losses during data transmission

- Smartphone Data Only: Only data recordable by smartphone will be used and transmitted on our network. We do not want to increase the complexity of our network by utilizing third-party equipment or data. By only exploiting smartphones data units we can also insure the optimization of our low-cost cooperative structure.

- Data Frequency: The data frequency of any recorded data will be limited to $1 \mathrm{~Hz}$ due to the data recording frequency of Android raw data measurements limited to $1 \mathrm{~Hz}$.

The goal for our collaborative smartphone-based network is to establish a cooperative structure taking advantages of smartphone's capabilities and volume in today's dense city centers. We envision a low-cost structure built around Android mobile phones positioning capabilities, scalable to the size of a city center. Our network must be accessible easily to multiple users.

\section{III.1.3 - Various cooperative solutions from the literature}

The literature review on cooperative positioning brought to light various techniques, methods and algorithms used in different fields of application. However, most of the techniques, exposed in these papers from the literature review, could not be applied to smartphone-based positioning in urban environment and/or do not meet the required criteria established for our collaborative network and listed in section III.1.2. Radar and laser ranging techniques [9] for example are not compatible with the philosophy of our network since they require additional third-party equipment. Other methods including the use of 3D city models [10][11] for urban positioning, 5G localization, and Bluetooth/Wi-Fi RSS based positioning techniques [12][13] tailored for indoor positioning do not comply with the specification of our network. Smartphone based collaborative work shows promising results. The implementation of a clustering algorithm has been tested in [14][15] that aim at detecting and bypassing measurements outliers shared between users. Moreover, Multiple authors [16][17] identified methods utilizing SoOP signals for range estimation between network's users.

\section{III.2 - Preliminary Testing}

Preliminary collaborative algorithms have been implemented in order to test cooperative techniques and methods studied. We picked a collaborative scenario that has been performed during the data collection campaign described in section II.1. The scenario selected is the one that we chose to call $\mathrm{C}$ on Figure 1. This collaborative scenario took place in the city center of Toulouse in a deep urban canyon. In this scenario, one smartphone (so called "adding user") is placed in an open sky environment being static. While the second smartphone (so called "aided user") is navigating around the position of the first smartphone in a constrained urban environment. On the left side of Figure 6, the trajectory of both smartphones is represented. The green dots represent the adding static user and is named user B. The blue dots show the dynamic-urban aided user, named user A, with the reference trajectory being shown in red.

We are working with real-life data recorded of both smartphones. We were able to reproduce this scenario by parking one of the vehicles on the top-open floor of a silo parking lot in the middle of the city center, while the second vehicle was navigating around the area.

Our testing support is a dual-PVT estimation algorithm that is able to estimate the positioning solution of two receiver simultaneously using Android raw data measurements. The graph on the right of Figure 6, shows the positioning error of user B compared to the reference trajectory without any collaboration from user A. This error evaluation corresponds to the foundation of our collaborative algorithm. The implementation of future cooperative algorithms will show the impact of the aiding user A on the positioning accuracy of user B compared to the reference trajectory.

Future collaborative work around this scenario would be the computation of the estimate of the range between two users described in [18]. We expect to see a significant increase of positioning accuracy with the use of aiding techniques and algorithms. 

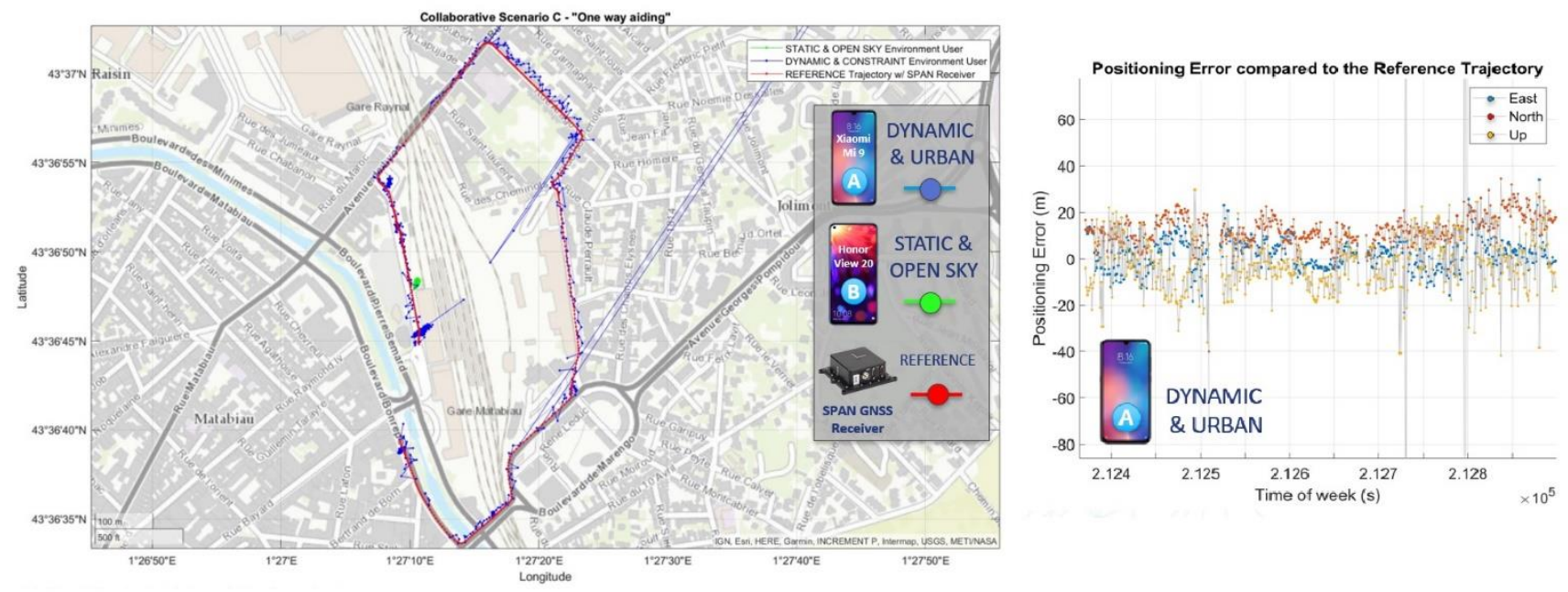

Figure 6: Left: Users A \& B Trajectory for Scenario C. - Right: Positioning Error of User A Compared to the Reference.

\section{CONCLUSIONS}

An assessment methodology describing various Android smartphones' GNSS behavior has been established. Describing key parameters for nominal conditions and analyzing Android GNSS raw data measurements in urban environment allowed us to better cope with Android embedded GNSS receivers "black box" processes. Studying multiple brands and models showed a clear difference in positioning performances between devices. Future smartphones' positioning capabilities and performance should be monitored thoroughly following the methodology presented in this paper. The examination of real data in an urban environment by recording Android GNSS raw data measurements revealed that smartphones can be used in a collaborative network system.

The creation and architecture of a smartphone-only collaborative network has been studied. In this paper we were able to define and characterize the base model for our low-cost cooperative cluster of smartphones' users. Limitations, hypothesis and an overall goal have been set in regards of the state-of-the-art review made on various collaborative works.

Future works will be focused on the implementation of innovative collaborative techniques in a smartphone-based collaborative network for tackling urban environment positioning constraints.

\section{ACKNOWLEDGMENTS}

The authors would like to thank ENAC researchers for their help during the data collection campaign. Jeremy VEZINET, Maxandre COULON, Paul THEVENON as well as Cedric ROUCH from CNES provided us with high-end GNSS receivers, multiple equipment and two research vehicles in the context of our smartphones testing study. Their involvement in this research work made our innovative and challenging urban data collection campaign possible. This study has been made in the context of my PhD thesis co-financed by CNES (French space agency) and Thalès Alenia Space.

\section{REFERENCES}

1. Chen B., Chengfa G., Yongsheng L. and Puyu S., "Real-time Precise Point Positioning with a Xiaomi Mi 8 Android Smartphone", Remote Sensors, Control, and Telemetry., 2019.

2. Pesyna K. M., Heath R. W. and Humphreys T. E., "Centimeter Positioning with a Smartphone-quality GNSS Antenna", GPS World, 2015.

3. Robustelli U., Baiocchi V. and Pugliano G., "Assessment of Dual Frequency GNSS Observations from a Xiaomi Mi 8 Android Smartphone and Positioning Performance Analysis", Green Communications in Smart City, 2019. 
4. GSA GNSS Raw Measurements Task Force, "Using GNSS Raw Measurements on Android Devices", White Paper, January 2018, https://www.gsa.europa.eu/system/files/reports/gnss_raw_measurement_web_0.pdf .

5. Lehtola V.V., Söderholm S., Koivisto M. and Montloin L., "Exploring GNSS Crowdsourcing Feasibility: Combinations of Measurements for Modeling Smartphone and Higher End GNSS Receiver Performance", Sensors, vol. 19, pp. 1-17, 2019. https://doi.org/10.3390/s19133018.

6. Verheyde T., Blais A., Macabiau C. and Marmet F-X., "Statistical Analysis of Android GNSS Raw Data Measurements in an Urban Environment for Smartphone Collaborative Positioning Methods," Session Al - International Navigation Conference (INC 2019), Presentation, Edinburgh 2019.

7. Verheyde T., Blais A., Macabiau C. and Marmet F-X., "Analyzing Android GNSS Raw Measurements Flags Detection Mechanisms for Collaborative Positioning in Urban Environment", 2020 International Conference on Localization and GNSS (ICL-GNSS), Tampere, Finland, 2020, pp. 1-6, doi: 10.1109/ICL-GNSS49876.2020.9115564.

8. Garello R., LoPresti L., Corraza G. E. and Samson J., "Peer-to-Peer Cooperative Positioning, Part I: GNSS-Aided Acquisition", Inside GNSS, pp. 55-63, 2012.

9. Gao Y., Liu S., Atia M. M. and Noureldin A., "INS/GPS/LIDAR Integrated Navigation System for Urban and Indoor Environments using Hybrid Scan Matching Algorithm", Sensors, vol. 15, no. 9, pp. 23 286-23 302, 2015.

10. Wang L., Groves P., and Ziebart M., "Urban Positioning on a Smartphone: Real-Time Shadow Matching Using GNSS and 3D City Models", Inside GNSS (Magazine), vol. 8, pp. 44.56, 112013.

11. Betaille D., Miquel S., Godan F. and Peyret F., “3D-city-model-aided GNSS Accurate Positioning with Integrity Provision using Simplified Geometry of Buildings", European Navigation Conference, Apr 2015, Bordeaux, France.

12. Guangyi G., Ruizhi C., Feng Y., Xuesheng P., Zuoya L., and Yuanjin P., "Indoor Smartphone Localization: A Hybrid WiFi RTT-RSS Ranging Approach”, IEEE Access. 7. 1-1. 10.1109/ACCESS.2019.2957753, 2019.

13. Matos D., Moreira A., and Meneses F., "Wi-Fi Fingerprint Similarity in Collaborative Radio Maps for Indoor Positioning", http://hdl.handle.net/1822/31236, Conference paper, 2014.

14. Liang H., Kim H. S., Tan H. and Yeow W., "Where am I? Characterizing and improving the localization performance of off-the-shelf mobile devices through cooperation," NOMS 2016 - 2016 IEEE/IFIP Network Operations and Management Symposium, Istanbul, 2016, pp. 375-382, doi: 10.1109/NOMS.2016.7502834.

15. Gioia C. and Borio D., "Android positioning: from stand-alone to cooperative approaches", Appl Geomat (2020). https://doi.org/10.1007/s12518-020-00333-4

16. Ta, V.-C., Dao, T.-K., Vaufreydaz, D. and Castelli, E., "Collaborative Smartphone-Based User Positioning in a MultipleUser Context Using Wireless Technologies”, Sensors 2020, 20, 405.

17. Gogoi N., Minetto A. and Dovis F., "On the Cooperative Ranging between Android Smartphones Sharing Raw GNSS Measurements”, 10.1109/VTCFall.2019.8891320, 2019.

18. Minetto A., Cristodaro C. and Dovis F., "A Collaborative Method for Positioning Based on GNSS Inter Agent Range Estimation," 2017 25th European Signal Processing Conference (EUSIPCO), Kos, 2017, pp. 2714-2718, doi: 10.23919/EUSIPCO.2017.8081704. 